\title{
First Retrospective Analysis of 448 Pediatric Cases of Kawasaki Disease of Two Cohorts from China and Russia
}

\author{
MD Bakradze ${ }^{1,2}$, AS Polyakova ${ }^{1}$, V.K. Tatochenkoํㅜ, AA Gusev ${ }^{1}$, VV Chernikov ${ }^{1}$, OV Komarova ${ }^{1}$, AG \\ Timofeeva ${ }^{1}$, AP Fisenko ${ }^{1}$, Wang Juyan ${ }^{3}$, Cheng Yibing ${ }^{4}$, Feng Yingjun ${ }^{4}$, Zhang Sheng ${ }^{3}$, He Gaitao ${ }^{3}$, Li \\ Xiaohong ${ }^{3}$, Stefan Bittmann ${ }^{5}$, Jiao Fuyong ${ }^{3 *}$ \\ ${ }^{1}$ National Medical Research Center for Children's Health, Federal state autonomous institution of the Russian Federation Ministry of Health, Moskau, Russia
}

${ }^{2}$ Sechenov First Moscow State Medical University, Russian Federation, Moskau, Russia

${ }^{3}$ Children's Hospital of Shaanxi Provincial People's Hospital, Xiatong University, China

${ }^{4}$ Department of Cardiology, Children's Hospital Affiliated to Zhengzhou University, China

${ }^{5}$ Department of Pediatrics, Ped Mind Institute, Germany

*Corresponding author: Jiao Fuyong, Children's Hospital of Shaanxi Provincial People’s Hospital, Xiatong University, China

To Cite This Article: Jiao Fuyong. First Retrospective Analysis of 448 Pediatric Cases of Kawasaki Disease of Two Cohorts from China and Russia. 2020 - 9(3). AJBSR.MS.ID.001384. DOI: 10.34297/AJBSR.2020.09.001384.

Received: 䟧 May 29, 2020; Published: 眥 June 25, 2020

\begin{abstract}
Kawasaki disease (KD) is an acute multisystemic vasculitis of unknown etiology that occurs in infants and children. Diagnosis is based on clinical criteria that include fever, exanthema, conjunctivitis, changes in the extremities, erythema of oral mucosa and lips and cervical lymphadenopathy. We investigated the clinical signs and therapy efficacy of patients with KD who admitted to our department. We reviewed 22 patients in Russia, with an age range of 2,5 months to 12 years. Moreover, 426 cases of children with KD admitted to the Children's Hospital of Shaanxi Provincial People's Hospital (China) from December 2013 to March 2018 were selected and compared. In case of hospitalization within 10 days of fever we reviewed incomplete KD in 5 patients (25\%). In 3 patients KD was finally diagnosed either after antibiotics had been ineffective or when other symptoms of KD emerged, in $70 \%$ of cases the diagnosis of KD was undoubted. There was also no doubt of diagnosis in all patients admitted to our department after 10 days of fever. $77 \%$ of patients recovered after one dose $(2 \mathrm{~g} / \mathrm{kg})$ of intravenous immunoglobulin (IVIG) and in $23 \%$ of cases it was necessary to insert the second dose. Clinicians should consider the KD diagnosis in young children with persistent inexplicable fever to start IVIG treatment within 10 days to prevent development of coronary aneurysms.
\end{abstract}

\section{Introduction}

The Kawasaki disease (KD) or mucous and skin lymphonodular syndrome - an acute general disease with primary damage of average and small arteries and development of a destructive and proliferative vasculitis. Clinically KD proceeds with fever, modifications of mucous membranes, skin, lymph nodes, possible defeat of coronary and other visceral arteries [1]. For the first time this disease is described by T. Kawasaki in Japan in 1967 [2]. Since 1961, he examined children with persistent fever and such symptoms as cervical lymphadenitis, conjunctivitis, rash, edema of brushes and feet with the subsequent peeling of fingers. This symptom complex did not match any other known disease. T. Kawasaki described about 50 cases, then this state had the name "febrile oculo-oro-kutaneo-acrodesquamative syndrome with not purulent cervical lymphadenitis". In 1992 the disease as independent nosologically unit was allocated in the most important reference book of the American pediatricians -"Nelson text book" and received a name of its discoverer - Kawasaki disease. Clinical guidelines for the diagnosis and treatment of Kawasaki syndrome have been developed in Russia [3].

\section{Patients and Methods}

\section{Russia}

In Office of Diagnostics and Recovery Treatment of National medical research center of children health (Russian Federation) we 
observed 22 children with KD. One of them was 12 years old, other children - aged from 2.5 up to 36 months (middle age - 12 months). The ratio of boys and girls was 1:1,2. In case of unclear diagnosis for children with persistent fever in addition to a standard complex of the examinations, conducted at fever without the infection site, ultrasound examination of heart and coronary vessels was conducted. In case of KD confirmation heart ultrasonography was carried out repeatedly after $10^{\text {th }}$ day of diseases.

\section{China}

A total of 426 cases of children with KD admitted to the Children's Hospital of Shaanxi Provincial People's Hospital at Xiatong University in Xian from December 2013 to March 2018 were selected. Among them, 17 patients were diagnosed with coronary artery aneurysm by echo-cardio-graphy (including the newly diagnosed children in our hospital and the children with coronary artery aneurysm diagnosed in other hospitals for further treatment in our hospital). Clinical data of KD children with coronary aneurysm were collected. The age, gender, major clinical manifestations, laboratory findings (including CRP, ESR, PLT), ultrasound examination results (including coronary artery aneurysm detection time and coronary artery involvement), IVIG treatment methods and effects were retrospectively recorded and analyzed in KD children with coronary artery aneurysm.

The diagnosis of $\mathrm{KD}$ is based on the diagnosis, treatment and long-term management guideline for Kawasaki disease issued by the American Heart Association in 2004 [3], that is, clinical diagnosis can be made with at least 5 of the 6 main clinical manifestations; if 5 items are not satisfied, but echocardiography or cardiovascular angiography confirms coronary artery aneurysm or coronary artery dilatation, except for other diseases at the same time complete KD can also be diagnosed. Unexplained fever for 5 days or more combined with 2 or 3 typical KD clinical features may be considered incomplete $\mathrm{KD}$. The diagnosis criteria for coronary artery aneurysm [4]. the ratio of the inner diameter of the coronary artery expansion segment to the adjacent segment is greater than 1.5 , among which, the inner diameter of the aneurysm $<5 \mathrm{~mm}$ is a small coronary artery aneurysm, $5-8 \mathrm{~mm}$ is a medium coronary artery aneurysm and $>8 \mathrm{~mm}$ is a giant coronary artery aneurysm.

\section{Epidemiological Aspects of Kawasaki Disease}

The disease of Kawasaki is widespread among babies and infants. The incidence considerably varies between ethnic groups. The incidence of KD is equal to 5-10 cases per 100,000 children in recent European researches, under 5 years old [4-7]. In the Asian countries it is reported about much higher level $[8,9]$. The highest incidence is registered in Japan; the last national research in 2012 reports about incidence - 265/100.000 children, under 5 years old and assumes that the KD level still increases. Most of patients are babies from 6 months to 5 years old, in spite of the fact that incidence cases also take place among children of elder age and adults. A ratio of men/women - about 1,5 to 1 [7]. In the USA about 3000 new cases of KD are annually described - frequency 20,8 per 100,000 children, younger than 5 years old (dispersion from 18,5 to 23,1 in different years). $76.8 \%$ from all KD cases were diagnosed at this age, the ratio of boys and girls was about 3:2 [10,11]. For the 15 -year period of observations in Irkutsk region the average level of KD incidence was only 6,6 cases per 100,000 children under 5 year old and 2,7 cases per 100,000 children under 17 year old. Thus, patients under 5 year old were $67,5 \%$ of the diseased, out of them $50,8 \%$ - under 3 year old. Infants bear KD most severely, in Russia their share is $23,5 \%$ [12].

According to an epidemiological study conducted in Russia (Irkutsk location) from 2005 to $2009 \mathrm{KD}$ was in all seasons of the year [13]. The disease was more often recorded in spring - $30.2 \%$ and in winter $-28 \%$, less often in autumn $-22.8 \%$ and in summer - 19\%. A separate analysis of the seasonal incidence of BC among boys and girls found that in boys the disease was also more often recorded in the winter-spring period, moreover, more cases were recorded in boys (44.5\%) from January to April than in girls (34\%). Among girls, a more pronounced increase in the incidence rate was found than among boys, in the autumn-winter season with the largest number of cases from October to December (girls - 32\%, boys $-17.7 \%$ ). An interesting fact is that the risk of $\mathrm{BC}$ in these months in girls is 2 times higher than in boys ( $R R=2.19,95 \%$-DI 1.29-3.72).

In February, 2.7 times fewer cases of $\mathrm{BC}$ were registered among girls than among boys. A decrease in the incidence of $\mathrm{BC}$ in the summer months is observed in patients of both sexes, slightly more pronounced in girls (girls - $20.7 \%$, boys - $17 \%$ ). The unambiguous opinion on the cause of KD development does not exist. In favor of infectious nature, general inflammatory symptoms, an increase in the level of inflammation markers, seasonality and epidemic outbreaks are said. In this regard Epstein-Barre's virus, retro and parvoviruses, streptococci, staphylococcus and also toxins were considered. Multiple viral infectious triggers have been suggested, including coxsackie virus, para influenza virus, respiratory syncytial virus, human metapneumovirus, chikungunya, and cytomegalovirus. The fact that KD is rare in children under 4 months could indicate passive maternal immunity, and the extreme rarity in adults - to reflect artificial immunity. The geographical sequence of incidence growth (Asia - Japan - Hawaii - the West bank of the USA could be explained by proliferation of the KD pathogens due to the influence of the atmospheric phenomena $[14,15]$.

In fact, two recent studies showed that up to about half of all KD patients had one of more respiratory viruses detected by PCR, but their etiological role is unproven $[16,17]$. Also, the possibility of a respiratory RNA virus has been suggested by ultrastructural studies of autopsy specimens $[18,19]$. Bacteria have also been suggested as the trigger of $\mathrm{KD}$, with research mainly focusing on bacterial $\mathrm{s}$ 
uperantigens. Superantigens produced by several bacteria are able to stimulate a large percentage of $\mathrm{T}$ cells by binding to the $\mathrm{V} \beta$ region of $\mathrm{T}$ cell receptors and so stimulate the production of proinflammatory cytokines. One study looking at five superantigens (streptococcal pyrogenic exotoxin (SPE)-A, C, G, and J, and toxic shock syndrome toxin-1 (TSST-1)), found these in 70\% of stool samples collected from acute KD patients as opposed to $27 \%$ in healthy controls visiting the same center for vaccinations [20]. Another study found significantly increased IgM antibodies against five superantigens (staphylococcal enterotoxin A, B, and C, TSST1, and SPE-A) [21]. Nevertheless, the role of superantigens in KD remains unclear. An immune response plays role in KD pathogenesis.

The encounter of a susceptible individual with the unknown agent probably leads to an (exaggerated) immune response involving innate and adaptive pathways. Multiple studies have been performed, both evaluating animal models and immune response in the peripheral blood as well as immune infiltration in the coronary arteries $[22,23]$. The general paradigm of the immune response is an imbalance between pro-inflammatory and antiinflammatory pathways. For example, regulatory $\mathrm{T}$ cells, a subset of $\mathrm{T}$ cells limiting inflammation, have been shown to be important in the vascular inflammation [24]. Also, the IL-1 signaling pathway is upregulated, with upregulated IL-1 pathway genes and increased IL-1 concentrations in peripheral blood of KD patients during the acute phase $[25,26]$. Recently, it has become clear that inflammasomes, multiproteins that are part of the innate immune system, are induced by the NLRP3 gene and promote the production of IL-1 $\beta$ and IL-18, play a role in KD [27]. In the coronary arteries, immune infiltration of the arterial wall with neutrophils, CD8+ cytotoxic T cells, Ig-A producing plasma cells, and macrophages have been found, accompanied by pro-inflammatory cytokines which may vary in proportion and contribution over time [28].

Genetics are considered to contribute to susceptibility to KD, and probably to CAA and response to treatment $[29,30]$. A number of genome-wide association studies (GWAS) have been performed [31-37]. Apart from the GWAS, multiple studies have identified specific single nucleotide polymorphisms (SNPs) in several genes. Most of these candidate genes have an immune regulatory function. Pathomorphologically, in KD arteries of average and small size are infected, involvement in process of the coronary arteries (CA) - up to $20 \%$ of cases is of particular importance. At autopsy in CA hypostasis of endothelial and smooth muscle cells with inflammatory infiltration of all 3 layers of a vascular wall is revealed. It leads to fragmentation of an inner elastic membrane with forming of aneurysms. Neutrophils in infiltrates quickly give way to macrophages, lymphocytes (mostly, CD8 cells) and plasmocytes, which produce A immunoglobulin in its turn. Fibrosis with the vessel intima proliferation at the recovering stage eventually leads to stenosis or full occlusion of its gleam [38].

\section{Clinical Aspects of Kawasaki Disease}

The clinical picture of Kawasaki disease conditionally is divided into 3 stages. The acute feverish stage lasts 1-2 weeks, and without appropriate treatment - up to 3-4 weeks that increases risk of coronary arteries disease [39]. It is characterized by persistent febrile fever, lasting more than 5 days, but most often without treatment - 2-3 weeks. Lesion of mucous membranes usually is characterized with not purulent conjunctivitis, expressed by an injection of conjunctiva vessels (in 95\% of cases) (more bulbar - episcleritis) (Figure 1). Cheilosis, changes of oral cavity and tongue' mucous membrane ("crimson-coloured" tongue), cracks of lips, which we observed at 15 children (75\%) (Figure 2), are also usually seen. Lesion of skin is usually followed with rash on body appearance, limbs, inguinal area.

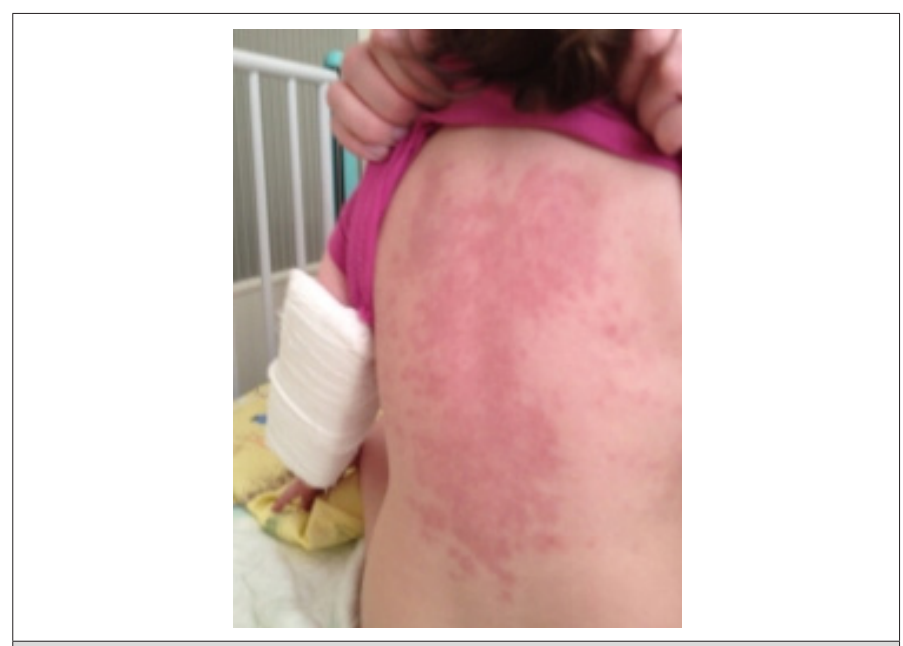

Figure 1: Typical rash in Kawasaki Disease on the back

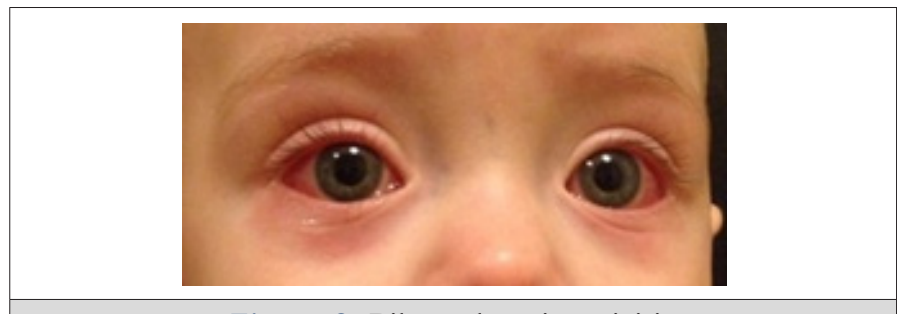

Figure 2: Bilateral conjunctivitis

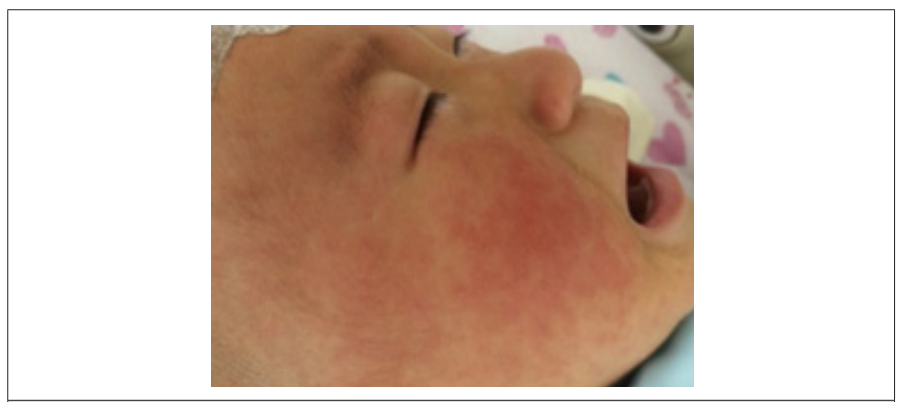

Figure 3: Typical rash in Kawasaki Disease in the face

The exanthema, sometimes drain, is mostly often characterized with papules and macules, though also urticarius and micropustulous elements could be seen. Bullas and vesicles were 
never described. We observed rash at $90 \%$ of patients, not always profuse, sometimes ephemeral; rash appeared on 2nd-6th days of a disease and died away in 2-4 days in all cases (Figure 3). Increase (unilateral) of front and cervical lymph nodes was diagnosed by us in half of cases, two children had a mask of purulent lymphadenitis with a local dermahemia and fluctuation. However, the puncture was not carried by these children, because existence of other KD signs allowed to prescribe immunoglobulin for intravenous administration, then changes of lymph nodes altogether with fever disappeared within 24 hours. Plasticity of brushes and, more often, feet, typical for KD (Figure 4) were observed at 12 patients in the first days of a disease, the morbidity of movements in joints and dermahemia above them was defined at 7 children. These symptoms were also stopped due to introduction of IVIG (Figure 5).
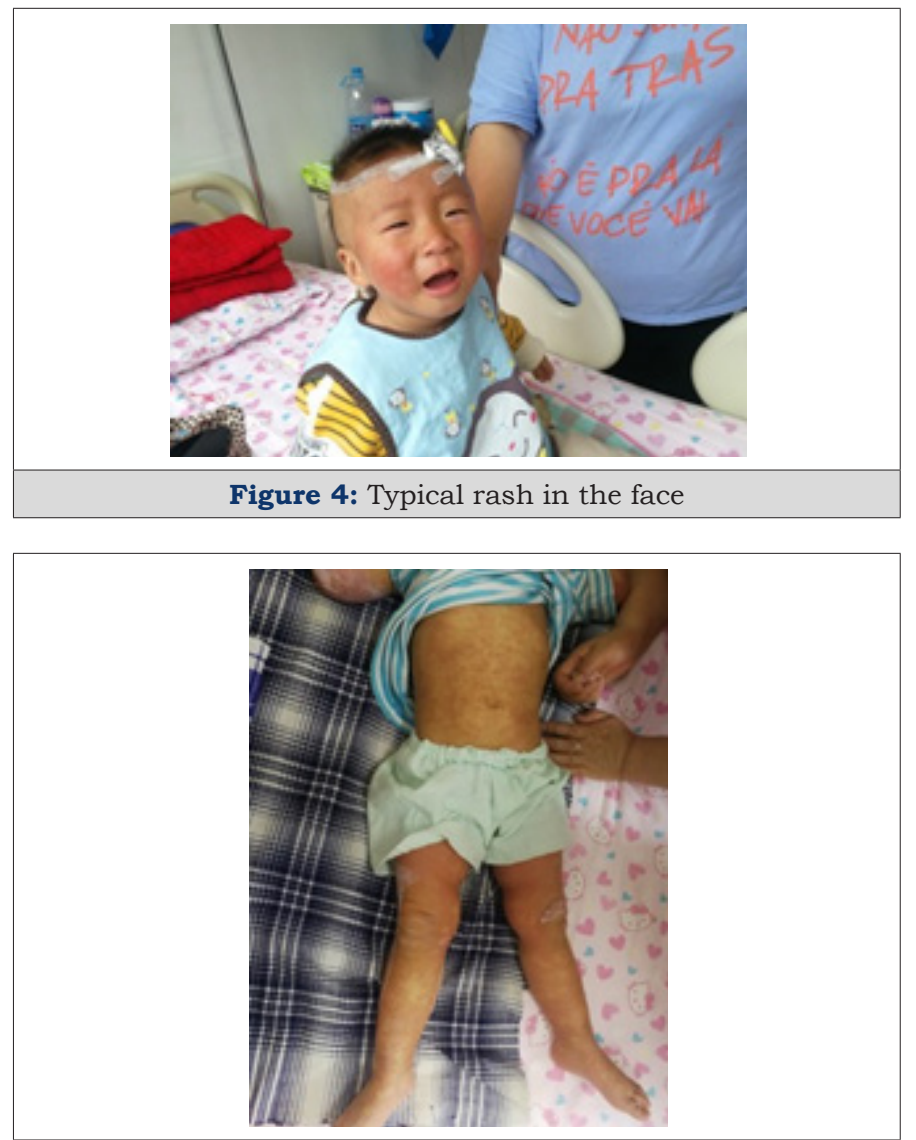

Figure 5: Typical rash in Kawasaki oft he whole body

For KD irritability of the patient is typical; the child is usually whining and inconsolable, it also could be a symptom of aseptic meningitis (the lymphocytic pleocytosis up to 25-100 in $1 \mu \mathrm{l}$ at the normal level of glucose and protein appears in cerebrospinal fluid). A typical clinical symptom at small children is reddening and consolidation of BCG injection place (patients with KD have a crossresponsiveness between proteins of thermal shock and T-cells). The subacute stage covers the 3-5th week of a disease, skin peeling, which begins with fingers of hands and legs and also in a groin, is typical for it with the background of the persistent fever. Since the end of the 2 nd disease week macrolaminar peeling was observed by us at $11(50 \%)$ children, the same - the marked peeling of a red border of lips. In 1-2 weeks after stopping of fever it is possible to observe cross deep lines on nails at some children (lines Beau). Recovery, as a rule, occurs in 6-10 weeks from disease appearance, all clinical symptoms disappear, SOE is gradually normalized. Changes from heart are manifested in the form of arrhythmias, heart noises («machine» noise), caused by regurgitation.

Echographic signs of a coronaritisis with a thickening of walls or expansion of coronary arteries' gleam and also ST-T changes of ischemic type on an ECG are registered more than at a half of patients; at a quarter of the patients, who were not receiving treatment in the KD acute period, symptoms of the postponed myocardial infarction reveal [40]. It is considered that forming of CA aneurysms happens on the 7-9th day of a disease. It is directly connected with destruction of their elastic frame. Aneurysms at the patients, who had KD, are found not only in CA, but in $2,2 \%$ of patients in the axillary, common and internal iliac, renal, subclavian, upper brychee, inner thoracic, femoral arteries [41]. We revealed signs of coronaritisis, such as thickening of walls and dilatation of coronary arteries, at 13 (65\%) patients, much more often at 3 of 4 children, during hospitalization after the 10th day of a disease. One child of 4 months, hospitalized on the $31^{\text {st }}$ day of fever, expansion of the left and right coronary arteries up to $4,5 \mathrm{~mm}$ was diagnosed. He responded to treatment of IVIG, and within 6 months the echographic picture was normalized. Without treatment in the acute period symptoms of heart failure, myocardium ischemia are often registered. According to the Russian authors, the heart failure (HF) develops in $56,3 \%$ of cases $(50 \%$ - HF of the $1-2$ nd functional class, $6,3 \%-$ the $3-4^{\text {th }}$ ).

In the same research myocardium dysfunction are met considerably more often at patients with CA dilatation, than without it $-78,3 \%$ and $43,8 \%$ respectively [18]. Huge aneurysms of CA (the internal diameter more than $8 \mathrm{~mm}$ ) are rarely met, however are fraught with risk of a cardiac tamponade, thrombosis or myocardial infarction. Other manifestations of KD. Tonsillitis, symptoms of pneumonia, gastroenterocolitis, liver failure, urinary and central nervous system failure are revealed quite often in a debut of KD. In these cases, especially in KD incomplete picture, naturally, fever are supposed to be like a bacterial infection in the beginning; but persistence of temperature after antibiotic prescription to the child with symptoms of an urine system or pneumonia infection allows to doubt the diagnosis, because it could delay KD diagnostics no more than for 2-3 days. The orchitis, pulmonary infiltrates, pleural exudate, haemo phagocytal syndrome [42-44] and also transient sensoneural deafness are considered as rare manifestations of a disease $[45,46]$.

The example of doubts in the diagnosis was when we faced the child, who came to hospital for the $3^{\text {rd }}$ day of fever with a leukecyturia, one kidney increase in the sizes, leukocytosis and 
increase in level of bacterial inflammation markers. Persistence of fever for the 3rd day of therapy with ceftriaxone, then meropeny and also appearance of generalized rash (in the beginning to be regarded as allergic), increase of sclerite, cheilitis and pastosity of feet expressiveness from the $5^{\text {th }}$ day of a disease made us to diagnose KD. Fever was stopped after IVIG introduction during the 6-7 $7^{\text {th }}$ day of a disease in a dose of $2 \mathrm{~g} / \mathrm{kg}$, the same day all other symptoms began to regress and macrolaminar peeling of hands' fingers appeared. Two more children, hospitalized till 10th day of diseases, the diagnosis of KD competed with suspicion of a severe bacterial infection with increase in inflammation markers. One of them had watery diarrhea. It made to the diagnosis in favor of bacterial intestinal infection, and second one - for lack of the infection site in favor of bacteremia. These suspicions were kind of confirmed by decrease in temperature after 36-48 hours of antibiotics therapy, however its new increase in a day with gaining of KD symptoms, became a reason for IVIG introduction, which gave fast effect.

\section{Laboratory Results (Moskau, Russia)}

The acute period reveal, as a rule, acute and inflammatory changes - a neutrophylic leukocytosis, increase in SOE, high level of C-jet protein and also normochromic normocytic anemia, but any of these tests are not pathognomonic for KD. Laboratory parameters usually come back to norm in 6-8 weeks. Data on pro-calcitonin differ a little: its level in the first days of a disease is high (3 and more $\mathrm{ng} / \mathrm{ml}$ ), but it is quickly normalized, so it usually comes to norm on the 2 nd week. Level of thrombocytes increases on the 1st week of a disease, considerably increases on the $2 \mathrm{nd}$, reaches peak on the 3rd week and gradually normalized on 6-10th week of a disease. Also other changes in the system of hemostasis, in particular, hyper aggregation of thrombocytes, exhaustion of a fibrinolytic system and also physiological anticoagulants appear [47].

It makes necessary their introduction to therapy. Predictors of KD severe state, increasing risk of cardiogenic complications, is increase in level of transaminases, general bilirubin, decrease in level of albumine and serumal sodium. Gammaglutamiltranspeptidase raises at $67 \%$ of patients [48]. In the clinical analysis of urine approximately in a third of cases the sterile proteinuria and proteinuria is revealed. The analysis of thousand of proteinaceous molecules showed that about 190 of them could be found in urine of children with KD. 2 proteins, associated with failure of endothelial and muscle cells, were identified: filamin $\mathrm{C}$ and immunoregulator meprin $\mathrm{A}$, which are considerably raised at the patients, who did not respond to starting therapy in comparison with the patients, who ceased to be in a fever after the first dose of IVIG. These indicators could be regarded as markers of disease activity, and increase of their level at children with KD incomplete form could theoretically be additional criterion of diagnosis [49].

\section{Laboratory Results (Xian, China)}

ESR: 1 week after admission $(50-100 \mathrm{~mm} / \mathrm{h} 4$ cases, $>100 \mathrm{~mm} / \mathrm{h}$
2 cases) and within 2 weeks of the disease $(50-100 \mathrm{~mm} / \mathrm{h} 3$ cases, $>100 \mathrm{~mm} / \mathrm{h} 1$ case), the rest were not examined. CRP: After admission, < 50mg/L 3 cases, 50-100mg/L 3 cases, >100mg/L 5 cases and the rest were not examined. At the time of discharge, < 50mg/L 10 cases and others were not examined. Among the 17 children with Kawasaki disease with coronary artery aneurysm, there was no significant difference in the degree of increase of ESR between 1 week and 2 weeks. After admission, 8 cases of CRP were significantly higher than normal, 6 cases were not examined, 10 cases were normal at discharge and 7 cases were not examined. The CRP level at discharge was significantly lower than that after admission.

Time of detection of coronary artery aneurysm in the course of disease: 1 case within 1 week, 12 cases within 2 weeks and 4 cases more than 1 month. Coronary artery aneurysms were usually detected within 2 weeks of the course of disease. Coronary artery involvement: there were 8 cases of bilateral coronary aneurysms, 4 cases of left coronary aneurysm alone, 5 cases of right coronary aneurysm alone. Bilateral coronary artery involvement was the most common.

\section{Diagnosis of Kawasaki Disease}

The diagnosis of KD is based on the presence of clinical features of persistent fever in combination with a polymorphous exanthema, cervical lymphadenopathy, non-purulent conjunctival injection, changes of the lips and oral cavity (including strawberry tongue, cracked lips, redness of the mucosae), and changes in extremities (swelling and redness of the palms, desquamation in the subacute phase) [50]. In the most recent American Heart Association (AHA) guidelines, persistent fever is classified as $\geq 5$ days, but in the presence of four or more symptoms, the diagnosis can be made with only 4 days of fever $[50,51]$. "Complete" $\mathrm{KD}$ is defined as fever and $\geq 4$ out of the 5 symptoms. It is important to appreciate that criteria may present successively instead of simultaneously. The AHA has created an algorithm to increase the possibility of "incomplete" KD in case $\leq 3$ criteria are present. This algorithm includes CA abnormalities on echocardiography and/ or laboratory abnormalities [50]. There is no diagnostic test for $\mathrm{KD}$, and the diagnosis may be delayed or overlooked. To improve diagnosis, multiple new biomarkers have been studied, but none has so far proved specific for KD [52]. Classification tools have been developed to aid in the differentiation between KD and other febrile illness, although the utility as a point-of-care diagnostic test remains unproven $[53,54]$.

\section{Russian Experience with Kawasaki with Patients}

In our examinations the diagnosis of KD was made in the $1^{\text {st }}$ day of hospitalization in $70 \%$ of the children, who came till $10^{\text {th }}$ day. The diagnosis of all children, hospitalized after $10^{\text {th }}$ day of disease, also did not make any doubts. Incomplete KD was diagnosed for 5 children (25\%). The girl of 3 months with fever within 9 days - 
the dermahemia over all interphalanx and to lesser extent - over metatarsal joints, disputable swelling of palms and feet and almost inevitable sclerite draw our attention. The diagnosis was made after identification of coronaritisis signs at heart ultrasonography. The dose of IVIG of $2 \mathrm{~g} / \mathrm{kg}$ did not make effect, after infusion expansion of right CA to 3,5 $\mathrm{mm}$ was seen. After the second dose of IVIG fever was stopped, right CA diameter decreased up to $2,4 \mathrm{~mm}$. The girl of 12 months for the 8th day of fever had only 2 signs out of 5 - swelling of brushes with a dermahemia over interphalangeal joints and expressed sclerite without signs of a coronaritisis. At introduction of IVIG in a dose of $2 \mathrm{~g} / \mathrm{kg}$ fever was stopped in the $1^{\text {st }}$ day.

The diagnosis of 5 months- girl was difficult with a periodic disease in the family anamnesis and carriage of the same gene mutation to distinguish. She came for the $32^{\text {nd }}$ day of fever; due to careful interrogation of mother we succeeded to know only about moderate conjunctivitis and light swelling of brushes in disease beginning; identification of left CA expansion up to 3,4 $\mathrm{mm}$ and right - up to 3,2 mm on ultrasonography helped to make the diagnosis. Introduction of IVIG in a dose of $4 \mathrm{~g} / \mathrm{kg}$ led knocks to apyrexia with the subsequent reduction of CA diameter up to $2,6 \mathrm{~mm}$ on the $3^{\text {rd }}$ day. The boy of 22 months came on the $18^{\text {th }}$ day of fever with sclerite and rash in the disease anamnesis. Identification of right CA expansion to 5,5 $\mathrm{mm}$ with consolidation of walls also confirmed diagnosis. Continuous $4 \mathrm{~g} / \mathrm{kg}$ of IVIG with stopping of fever for 2 days, after which fever and sclerite came back. Pulsotherapy with methylprednisolonum in a dose of $30 \mathrm{mg} / \mathrm{kg}$ within 3 days gave positive effect with reduction of CA diameter up to $3 \mathrm{~mm}$ and normalization of hematologic indicators.

In the next 2 weeks the sclerite and subfebrile condition up to $37,7^{\circ} \mathrm{C}$ with a further absolute recovery periodically appeared. The girl of 1 year with a picture of retropharyngeal abscess (fever, morbidity at the movements of the head, lockjaw) and hypoechoic (20-30 pieces of $\mathrm{N}$ ), lenticular, not accumulating contrastexcrescence in retropharyngeal area, revealed in computer tomography. The lack of pus during opening the place of hypostasis on throat back wall helped to suspect KD and persistence of temperature despite antibacterial therapy. Besides, from mother's interrogation we succeeded to find out existence of a one-day injection of scleras and ephemeral rash in the 1-2nd days of a disease. Introduction of IVIG stopped fever, and on the 10th day peeling of fingers appeared. We found the description of the few similar observations in literature [55]. Incomplete KD meets more often in infants; therefore, as well as foreign authors, we recommend echocardiography carrying out to all children with unclear fever and laboratory markers of inflammation $[39,56]$.

\section{Differential Diagnosis}

A number of diseases with exanthema and changes of joints (the infection, which are followed by exanthema, syndromes of toxic shock and "the scalded skin", juvenile pseudorheumatism, Stephens-Johnson's syndrome, etc.) has signs, similar to KD. Part of them is excluded according to clinical data even with persistent fever less than 5 days (including - effect on antibiotic therapy). At infectious mononucleosis in $10-15 \%$ of cases there is maculopapular rash, but fever is kept usually less than a week and laboratory data help with the diagnosis verification. StephensJohnson's syndrome differs from KD with violent and erosive elements. Juvenile idiopathic arthritis is quite often shown only by fever, generalized lymphadenopathy and spotty pale "flying" rash within several weeks. This diagnosis, as well as a nodular polyarteritis, must be meant at patients with suspicion on KD, whom were carried out treatment with an adequate dose of IVIG without effect. We observed a similar picture at the infant, that altogether with the instruction on "possible changes of coronary arteries walls" on heart ultrasonography, made to do IVIG, which did not give the expected improvement; further juvenile idiopathic arthritis was diagnosed for the child.

\section{Treatment and Prognosis of Kawasaki Patients in Russia}

Starting therapy of both full and incomplete KD assumes introduction of $2 \mathrm{~g} / \mathrm{kg}$ of IVIG given over 8-12 h [57]. Treatment with IVIG significantly reduces the incidence of CAA [58]. IVIG is preferably given within the first 10 days after disease onset [50]. Acetylsalicylic acid (as anti-inflammatory and antiaggregant drug) in a dose of $80-100 \mathrm{mg} / \mathrm{kg} /$ day in 4 receptions. Apart from IVIG, high-dose aspirin is advised by the AHA, although evidence for further risk reduction for CAA is lacking [58,59]. Additional prescription of anticoagulants (warfarin, dipyridamolum) could be required by children with huge coronary aneurysms. In Japan there is an experience of treatment by smaller doses of acetylsalicylic acid - 30-50 mg/kg/day - for the purpose of hepatotoxic effect prevention $[58,60]$. Most of children respond to IVIG introduction with fever stopping and considerable improvement of health within the first 24 hours, at the same time the risk of vessels and heart failure, forming of aneurysms authentically decreases (from $20 \%$ to $5 \%$ ). About $10-20 \%$ of the patients, who received IVIG course, have repeated fever [7].

In that case immunoglobulin is given repeatedly in the same dose $(2 \mathrm{~g} / \mathrm{kg})$. In Japan, risk-scores have been developed to identify patients with a higher risk of IVIG resistance [61- 63]. Unfortunately, these risk-scores do not perform adequately in Western, ethnically mixed, and in Chinese populations [64-68]. A possible method to decrease IVIG resistance is to intensify the initial treatment. A recent meta-analysis showed a beneficial effect of adding corticosteroids to the initial treatment with IVIG, yet this effect was only found in Japanese studies and not in two studies conducted in the USA [69]. Only in case of inefficiency of IVIG repeated therapy the question of infliximabum prescription is 
considered. Though there are data on its efficiency as starting drug at KD treatment [70]. Patients are considered to be resistant to IVIG, if in 2 days and more after an initial dose it was necessary to give a repeated dose, methylprednisolonum, rituximab or infliximabum. Resistant KD, according to researches, is met in $16,3 \%$ of cases with ratio between clinics of 8,0-26,8\%. More often cases of resistance are registered among the Afro-Americans [71]. After normalization of temperature, the AHA advises ongoing aspirin in a low dose until no evidence of CA dilation are present at by 4 to 6 weeks after the acute illness [50]. If CAA are present and persisting around that time, aspirin is continued as anti-thrombotic therapy.

In case of large (around a z-score $\geq 10$ ) or complex abnormalities, additional anticoagulation therapy should be administered to prevent clotting due to turbulence in these pro-coagulatory large coronary artery lesions [50,72]. In case of possible inactivation of live vaccines after IVIG treatment, vaccination against measles, epidemic parotitis and rubella must be postponed for 11 months. There is also a risk of development of Ray syndrome in children, who receive acetylsalicylic acid for a long time, especially at flu or chicken pox disease. In case of the child contact or disease of these infections temporary cancellation of acetylsalicylic acid is possible, and to children with existence of aneurysm - prescription of dipyridamolum on this time. KD is the leading reason of heart acquired diseases at children, increasing risk of development of coronary heart disease and myocardial infarction at young age. Male, aged under 1 year old, persistent fever and also KD recurrence are to be predictively adverse factors of CA aneurysm development. Thrombocytopenia, hypoalbuminemia and also low level of hemoglobin, G-class immunoglobulins are predictors of KD severe current with a possible outcome in CA aneurysm forming $[2,39]$.

In case of KD timely adequate treatment, for example in the USA, lethality is less than $0,01 \%$. Follow-up observation of $546 \mathrm{KD}$ cases showed that 5 of them died. Cause of death in 1 case was CA failure, in 1 case - sudden death without symptoms of thrombosis or myocardial infarction, in 1 case - an acute coronary syndrome as a result of aneurysm thrombosis, in 2 - other options of an acute coronary syndrome. In $50 \%$ of cases death came within a month after an acute state [73]. Approximately $2 \%$ of patients have KD recurrence in several months and even years after the 1st disease. Among the children, observed by us, one 6-month-old child had KD recurrence in 2 months after the 1st episode, treated in other hospital. The girl came on the 1st day of fever with vivid clinical manifestations of a disease and expansion of coronary vessels. Fever was stopped after the 1st IVIG dose with further regression of clinical symptoms and normalization of ultrasonic changes.

Nowadays many researches is devoted to studying of KD role in development of periodic feverish syndromes and some autoimmune diseases [74]. All children with KD, observed by us, were treated by IVIG, in $77 \%$ of cases we got lasting effect after single dose of $2 \mathrm{~g} / \mathrm{kg}$ in the form of temperature normalization, considerable improvement of state. In $23 \%$ of cases (5 children) it was necessary to repeat IVIG therapy, it significantly raised the price of treatment. Use of acetylsalicylic acid in a dose of $80-100 \mathrm{mg} / \mathrm{kg} /$ day in 4 receptions did not cause by-effects in our observations, as well as its further prolonged use in a low dose (3-5 mg/kg/day).

\section{Conclusion}

We had a possibility of 22 children with KD examination, both on the earliest and at late stages of a disease. All these children were hospitalized with one persistent complaint - persistence of fever, which was not stopped before specific treatment. The lack of several typical symptoms, which after all appeared in later terms at most of patients, was one of the main difficulties of $\mathrm{KD}$ diagnostics at early stages of a disease before emergence of coronaritisis' signs. Besides, it was possible to find out in parents' interrogation existence of some typical symptoms of a disease, which appeared at the very beginning of a disease and disappeared until hospitalization. Measures on timely diagnostics and adequate therapy of KD, according to the international standards and recommendations, include the focused attitude towards children with the persistent fever, which is especially not stopped with antibiotics, structured interrogation of parents about existence of KD symptoms at the very beginning of a disease and timely qualified ultrasonic examination of coronary arteries.

After discharge from a hospital long follow-up observation with control of heart ultrasonography and hematologic indicators for patients is carried out.

\section{Acknowledgement}

We like to thank Stefan Bittmann, MD MA, to work on correction, formatting and critical review of the manuscript. Stefan Bittmann and Jiao Fuyong work on a research basis in different fields of pediatric research in cooperation between China and Germany.

\section{Reference}

1. The Merck Manual (2015) Guide to medicine. Diagnosis and treatment. Moscow: litter .

2. Kawasaki T (1967) Acute febrile mucocutaneous syndrome with lymphoid involvement with specific desquamation of the fingers and toes in children. Arerugi 16(3): 178-222.

3. Baranov AA, Namazova Baranova LS, Tatochenko VK, Basargina EN, Revunenkov GV, et al. (2015) Federal clinical guidelines for providing medical care to children with mucocutaneous lymphonodular syndrome [Kawasaki]. Moscow.

4. Hall GC, Tulloh LE, Tulloh RM (2016) Kawasaki disease incidence in children and adolescents: an observational study in primary care. Br J Gen Pract 66(645): 271-276.

5. Jakob A, Whelan J, Kordecki M, Berner R, Stiller B, et al. (2016) Kawasaki disease in Germany: a prospective, population-based study adjusted for underreporting. Pediatr Infect Dis J 35(2): 129-134. 
6. Sanchez Manubens J, Anton J, Bou R, Iglesias E, Calzada-Hernandez J, et al. (2016) Incidence, epidemiology and clinical features of Kawasaki disease in Catalonia, Spain. Clin Exp Rheumatol 34(3 Suppl 97): 139-144.

7. Tacke CE, Breunis WB, Pereira RR, Breur JM, Kuipers IM, et al. (2014) Five years of Kawasaki disease in the Netherlands: a national surveillance study. Pediatr Infect Dis J 33(8): 793-797.

8. Chen JJ, Ma XJ, Liu F, Yan WL, Huang MR, et al. (2016) Epidemiologic features of Kawasaki disease in Shanghai from 2008 through 2012. Pediatr Infect Dis J 35(1): 7-12.

9. Ha S, Seo GH, Kim KY, Kim DS (2016) Epidemiologic study on Kawasaki disease in Korea, 2007-2014: based on health insurance review \& assessment service claims. J Korean Med Sci 31(9): 1445-1449.

10. Robert M Kliegman MD, Bonita F Stanton MD, Joseph W St Geme III MD, Nina F Schor MD, Richard E. Behrman MD, et al. (2011) Nelson textbook of pediatrics. Elsevier saunders, 19-th edition.

11. Holman Robert C, Belay Ermias D, Christensen Krista Y, Folkema Arianne M, Steiner Claudia A, et al. (2010) Hospitalizations for Kawasaki syndrome among children in the United States, 1997-2007. Pediatr Infect Dis J 29(6): 483-488.

12. Bregel LV, Soldatova TA, Subbotin VM, Kawasaki T, Yanagawa H, et al (2012) Epidemiological features of Kawasaki disease in Russia and Japan. Abstracts of the VII All-Russian Congress on Pediatric Cardiology. Moscow.

13. Bregel LV, Subbotin VM, Soldatova TA, Belozerov Yu M, Boyko TV, et al. (2011) Epidemiological features of Kawasaki disease in the Irkutsk region: the results of many years of observation. Pediatrics 90(5): 49-53.

14. Kao AS, Getis A, Brodine S, Burns JC (2008) Spatial and temporal clustering of Kawasaki syndrome cases. Pediatr Infect Dis J 27(11): 981-985.

15. Makino N, Nakamura Y, Yashiro M, Ae R, Tsuboi S, et al. (2015) Descriptive epidemiology of Kawasaki disease in Japan, 2011-2012: from the results of the 22nd nationwide survey. J Epidemiol 25(3): 239-245.

16. Chang LY, Lu CY, Shao PL, Lee PI, Lin MT, et al. (2014) Viral infections associated with Kawasaki disease. J Formos Med Assoc 113(3): 148-154.

17. Turnier JL, Anderson MS, Heizer HR, Jone PN, Glode MP, et al. (2015) Concurrent respiratory viruses and Kawasaki disease. Pediatrics 136(3): 609-614.

18. Rowley AH, Baker SC, Orenstein JM, Shulman ST (2008) Searching for the cause of Kawasaki disease-cytoplasmic inclusion bodies provide new insight. Nat Rev Microbiol 6(5): 394-401.

19. Rowley AH, Baker SC, Shulman ST, Rand KH, Tretiakova MS, et al. (2011) Ultrastructural, immunofluorescence, and RNA evidence support the hypothesis of a "new" virus associated with Kawasaki disease. J Infect Dis 203(7): 1021-1030.

20. Suenaga T, Suzuki H, Shibuta S, Takeuchi T, Yoshikawa N, et al. (2009) Detection of multiple superantigen genes in stools of patients with Kawasaki disease. J Pediatr 155(2): 266-270.

21. Matsubara K, Fukaya T, Miwa K, Shibayama N, Nigami H, et al. (2006) Development of serum IgM antibodies against superantigens of Staphylococcus aureus and Streptococcus pyogenes in Kawasaki disease. Clin Exp Immunol 143(3): 427-434.

22. Hui-Yuen JS, Duong TT, Yeung RS (2006) TNF-alpha is necessary for induction of coronary artery inflammation and aneurysm formation in an animal model of Kawasaki disease. J Immunol 176(10): 6294-6301.

23. Yeung RS (2010) Kawasaki disease: update on pathogenesis. Curr Opin Rheumatol 22(5): 551-560.

24. Franco A, Touma R, Song Y, Shimizu C, Tremoulet AH, et al. (2014) Specificity of regulatory $\mathrm{T}$ cells that modulate vascular inflammation. Autoimmunity 47(2): 95-104.
25. Hoang LT, Shimizu C, Ling L, Naim AN, Khor CC, et al. (2014) Global gene expression profiling identifies new therapeutic targets in acute Kawasaki disease. Genome Med 6(11): Pp541.

26. Suzuki H, Uemura S, Tone S, Iizuka T, Koike M, et al. (1996) Effects of immunoglobulin and gamma-interferon on the production of tumour necrosis factor-alpha and interleukin-1 beta by peripheral blood monocytes in the acute phase of Kawasaki disease. Eur J Pediatr 155(4): 291296.

27. Alphonse MP, Duong TT, Shumitzu C, Hoang TL, McCrindle BW, et al. (2016) Inositol-triphosphate 3-kinase $C$ mediates inflammasome activation and treatment response in Kawasaki disease. J Immunol 197(9): 3481-3489.

28. Brown TJ, Crawford SE, Cornwall ML, Garcia F, Shulman ST, et al. (2001) CD8 T lymphocytes and macrophages infiltrate coronary artery aneurysms in acute Kawasaki disease. J Infect Dis 184(7): 940-943.

29. Onouchi Y (2012) Genetics of Kawasaki disease: what we know and don't know. Circ J 76(7): 1581-1586.

30. Yoon KL (2015) Update of genetic susceptibility in patients with Kawasaki disease. Korean J Pediatr 58(3): 84-88.

31. Burgner D, Davila S, Breunis WB, Ng SB, Li Y, et al. (2009) A genome-wide association study identifies novel and functionally related susceptibility loci for Kawasaki disease. PLoS Genet 5(1): Pp1000319.

32. Kim JJ, Hong YM, Sohn S, Jang GY, Ha KS, et al. (2011) A genome-wide association analysis reveals $1 \mathrm{p} 31$ and $2 \mathrm{p} 13.3$ as susceptibility loci for Kawasaki disease. Hum Genet 129(5): 487-495.

33. Kuo HC, Li SC, Guo MM, Huang YH, Yu HR, et al. (2016) Genome-wide association study identifies novel susceptibility genes associated with coronary artery aneurysm formation in Kawasaki disease. PLoS One 11(5): Pp0154943.

34. Lee YC, Kuo HC, Chang JS, Chang LY, Huang LM, et al. (2012) Two new susceptibility loci for Kawasaki disease identified through genome-wide association analysis. Nat Genet 44(5): 522-525.

35. Onouchi Y, Gunji T, Burns JC, Shimizu C, Newburger JW, et al. (2008) ITPKC functional polymorphism associated with Kawasaki disease susceptibility and formation of coronary artery aneurysms. Nat Genet 40(1): $35-42$.

36. Onouchi Y, Ozaki K, Burns JC, Shimizu C, Terai M, et al. (2012) A genome-wide association study identifies three new risk loci for Kawasaki disease. Nat Genet 44(5) :517-521

37. Thai FJ, Lee YC, Chang JS, Huang LM, Huang FY, et al. (2011) Identification of novel susceptibility loci for Kawasaki disease in a Han Chinese population by a genome-wide association study. PLoS One 6(2): Pp16853.

38. Burns JC (2007) The riddle of Kawasaki disease. N Engl J Med 356(7): 659-661.

39. Jane W Newburger, Masato Takahashi, Michael A Gerber, Michael H Gewitz, Lloyd Y Tani, et al. (2004) Diagnosis, Treatment, and Long-Term Management of Kawasaki Disease: A Statement for Health Professionals From the Committee on Rheumatic Fever, Endocarditis, and Kawasaki Disease, Council on Cardiovascular Disease in the Young, American Heart Association. Pediatrics 110(17): 2747-3771.

40. Loginova MS, Bregel LV, Subbotin VM (2012) Cardiac manifestations of Kawasaki disease. Abstracts of the VII All-Russian Congress on Pediatric Cardiology. Moscow.

41. Kato H, Sugimura T, Akagi T, Sato N, Hashino K, et al. (1996) Long-term consequences of Kawasaki disease. A 10- to 21 year follow-up study of 594 patients. Circulation 94(6): 1379-1385.

42. Freeman AF, Crawford SE, Finn LS, Lopez-Andreu JA, Ferrando-Monlen S, et al. (2003) Inflammatory pulmonary nodules in Kawasaki disease. Pediatr Pulmonol 36(2): 102-106. 
43. Uziel Y, Hashkes PJ, Kassem E, Gottesman G, Wolach B (2003) “Unresolving pneumonia" as the main manifestation of atypical Kawasaki disease. Arch Dis Child 88(10): 940-942.

44. Palazzi DL, McClain KL, Kaplan SL (2003) Hemophagocytic syndrome after Kawasaki disease. Pediatr Infect Dis J 22(7): 663-666.

45. Sundel RP, Cleveland SS, Beiser AS, Newburger JW, McGill T, et al. (1992) Audiologic profiles of children with Kawasaki disease. Am j otol 13(6): 512-515.

46. Knott PD, Orloff LA, Harris JP, Novak RE, Burns JC, et al. (2001) Sensorineural hearing loss and Kawasaki disease: a prospective study. Am J Otolaryngol 22(5): 343-348.

47. Bregel LV, Subbotin VM, Nikitina EA (2004) Coronary complications and disorders in the hemostatic system in Kawasaki disease. Russian Bulletin of Perinatology and Pediatrics 17: p9.

48. Ting EC, Capable EV, Billman GF, Lavigne JE, Matsubara T, et al. (1998) Elevated gamma-glutamyltransferase concentrations in patients with acute Kawasaki disease. Pediatr infect dis J 17(5): 431-432.

49. Kentsis A, Shulman A, Ahmed S, Brennan E, Monuteaux MC, et al. (2013) Urine proteomics for discovery of improved diagnostic markers of Kawasaki disease. EMBO Mol Med 5(2): 210-220.

50. McCrindle BW, Rowley AH, Newburger JW, Burns JC, Bolger AF, et al. (2017) Diagnosis, treatment, and long-term management of kawasaki disease: a scientific statement for health professionals from the American Heart Association. Circulation 135(17): 927-999.

51. Phuong LK, Bonetto C, Buttery J, Pernus YB, Chandler R, et al. (2016) Kawasaki disease and immunisation: standardised case definition \& guidelines for data collection, analysis. Vaccine 34(51): 6582-6596.

52. Parthasarathy P, Agarwal A, Chawla K, Tofighi T, Mondal TK (2015) Upcoming biomarkers for the diagnosis of Kawasaki disease: a review. Clin Biochem 48(16-17): 1188-1194.

53. Hao S, Jin B, Tan Z, Li Z, Ji J, et al. (2016) A classification tool for differentiation of Kawasaki disease from other febrile illnesses. J Pediatr 176 114-120.

54. Ling XB, Kanegaye JT, Ji J, Peng S, Sato Y, et al. (2013) Point-of-care differentiation of Kawasaki disease from other febrile illnesses. J Pediatr 162(1): 183-188

55. Kato H, Kanematsu M, Kato Z, Teramoto T, Kondo N, et al. (2012) Computed tomographic findings of Kawasaki disease with cervical lymphadenopathy. J Comput Assist Tomogr 36(1): 138-142.

56. Baranov AA, Tatochenko VK, Bakradze MD (2017) Febrile child. Diagnostic and treatment protocols. Moscow, Pediatric 320 pp.

57. Newburger JW, Takahashi M, Beiser AS, Burns JC, Bastian J, et al. (1991) A single intravenous infusion of gamma globulin as compared with four infusions in the treatment of acute Kawasaki syndrome. N Engl J Med 324(23): 1633-1639.

58. Terai M, Shulman ST (1997) Prevalence of coronary artery abnormalities in Kawasaki disease is highly dependent on gamma globulin dose but independent of salicylate dose. J Pediatr 131(6): 888-893.

59. Durongpisitkul K, Gururaj VJ, Park JM, Martin CF (1995) The prevention of coronary artery aneurysm in Kawasaki disease: a meta-analysis on the efficacy of aspirin and immunoglobulin treatment. Pediatrics. 96(6): 1057-1061.
60. Williams RV, Minich LL, Tani LY (2001) Pharmacological therapy for patients with Kawasaki disease. Pediatr drugs 3(9): 649-660.

61. Egami K, Muta H, Ishii M, Suda K, Sugahara Y, et al. (2006) Prediction of resistance to intravenous immunoglobulin treatment in patients with Kawasaki disease. J Pediatr 149(2): 237-240.

62. Kobayashi T, Inoue Y, Takeuchi K, Okada Y, Tamura K, et al. (2006) Prediction of intravenous immunoglobulin unresponsiveness in patients with Kawasaki disease. Circulation 113(22): 2606-2612.

63. Sano T, Kurotobi S, Matsuzaki K, Yamamoto T, Maki I, et al. (2007) Prediction of non-responsiveness to standard high-dose gamma-globulin therapy in patients with acute Kawasaki disease before starting initial treatment. Eur J Pediatr 166(2): 131-137.

64. Davies S, Sutton N, Blackstock S, Gormley S, Hoggart CJ, et al. (2015) Predicting IVIG resistance in UK Kawasaki disease. Arch Dis Child 100(4): 366-368.

65. Loomba RS, Raskin A, Gudausky TM, Kirkpatrick E (2016) Role of the Egami score in predicting intravenous immunoglobulin resistance in Kawasaki disease among different ethnicities. Am J Ther 23(6): 1293-1299.

66. Sanchez Manubens J, Anton J, Bou R, Iglesias E, Calzada Hernandez J, et al. (2016) Role of the Egami score to predict immunoglobulin resistance in Kawasaki disease among a Western Mediterranean population. Rheumatol Int 36(7): 905-910.

67. Sleeper LA, Minich LL, McCrindle BM, Li JS, Mason W, et al. (2011) Evaluation of Kawasaki disease risk-scoring systems for intravenous immunoglobulin resistance. J Pediatr 158(5): 831-835.

68. Song R, Yao W, Li X (2017) Efficacy of four scoring systems in predicting intravenous immunoglobulin resistance in children with Kawasaki disease in a children's hospital in Beijing, North China. J Pediatr 184: 120-124.

69. Chen S, Dong Y, Kiuchi MG, Wang J, Li R, et al. (2016) Coronary artery complication in Kawasaki disease and the importance of early intervention: a systematic review and meta-analysis. JAMA Pediatr 170(12): 1156-1163.

70. Tremolet AH, Jain S, Jaggi P, Jimenez Fernandez S, Pancheri JM, et al (2014) Infliximab for intensification of primary therapy for Kawasaki disease: a phase 3 randomised, double-blind, placebo-controlled trial. Lancet 383(9930): 1731-1738.

71. Moffett BS, Syblik D, Denfield S, Altman C, Tejtel-Sexson K (2015) Epidemiology of immunoglobulin resistant kawasaki disease: results from a large, national database. Pediatr Cardiol 36(2): 374-378.

72. Newburger JW, Takahashi M, Burns JC (2016) Kawasaki disease. J Am Coll Cardiol 67(14): 1738-1749.

73. Holve TJ, Patel A, Chau Q, Marks AR, Meadows A, et al. (2014) Long-term cardiovascular outcomes in survivors of Kawasaki disease. Pediatrics 133(2): 305-311.

74. Broderick L, Adriana H Tremoulet, Jane C Burns, John F Bastian, Hal M (2011) Reccurent fever syndromes in patients after recovery from Kawasaki syndrome. Pediatrics 127(2): 489-493.

75. Singh S, Sharma A, Jiao FY (2016) Kawasaki Disease: Issues in Diagnosis and Treatment--A Developing Country Perspective. Indian J Pediatr 83(2): 140-145.

76. Jiao FY, Yan XH, Wu XF (2016) The Xi'an Experience of Kawasaki Disease-Lessons Learnt over 5 years. Indian J Pediatr 83(10): 1195-1196. 